\title{
Towards tailored radiopeptide therapy
}

\author{
Piotr Radojewski • Rebecca Dumont • Nicolas Marincek • \\ Philippe Brunner • Helmut R. Mäcke • Jan Müller-Brand • \\ Matthias Briel • Martin A. Walter
}

Received: 9 January 2015 / Accepted: 24 February 2015 /Published online: 20 March 2015

(C) Springer-Verlag Berlin Heidelberg 2015

\begin{abstract}
Purpose Somatostatin receptor-targeted radiopeptide therapy is commonly performed using single radioisotopes. We evaluated the benefits and harms of combining radioisotopes in radiopeptide therapy in patients with neuroendocrine tumor.

Methods Using multivariable-adjusted survival analyses and competing risk analyses we evaluated outcomes in patients with neuroendocrine tumor receiving ${ }^{90} \mathrm{Y}$-DOTATOC, ${ }^{177} \mathrm{Lu}$-DOTATOC or their combination.

Results ${ }^{90}$ Y-DOTATOC plus ${ }^{177}$ Lu-DOTATOC treatment was associated with longer survival than ${ }^{90} \mathrm{Y}$-DOTATOC (66.1 vs. 47.5 months; $n=1,358 ; p<0.001)$ or ${ }^{177}$ Lu-DOTATOC alone (66.1 vs. 45.5 months; $n=390 ; p<0.001$ ). ${ }^{177}$ Lu-DOTATOC was associated with longer survival than ${ }^{90} \mathrm{Y}$-DOTATOC in patients with solitary lesions (HR 0.3 , range $0.1-0.7 ; n=153$;
\end{abstract}

Electronic supplementary material The online version of this article (doi:10.1007/s00259-015-3030-9) contains supplementary material, which is available to authorized users.

\footnotetext{
P. Radojewski $\cdot$ N. Marincek $\cdot$ M. A. Walter $(\bowtie)$

Institute of Nuclear Medicine, University Hospital Bern,

3010 Bern, Switzerland

e-mail:m.a.walter@gmx.net

R. Dumont $\cdot$ N. Marincek · P. Brunner • J. Müller-Brand •

M. A. Walter

Institute of Nuclear Medicine, University Hospital Basel,

Basel, Switzerland

R. Dumont

Department of Radiology, David Geffen School of Medicine, UCLA, Los Angeles, CA, USA

H. R. Mäcke

Division of Radiological Chemistry, University Hospital Basel, Basel, Switzerland

\section{Briel}

Basel Institute for Clinical Epidemiology and Biostatistics, University Hospital Basel, Basel, Switzerland
}

$p=0.005$ ), extrahepatic metastases (HR 0.5, range $0.3-0.9$; $n=256 ; p=0.029$ ) and metastases with low uptake (HR 0.1, range $0.05-0.4 ; n=113 ; p=0.001) .{ }^{90} \mathrm{Y}$-DOTATOC induced higher hematotoxicity rates than combined treatment $(9.5 \%$ vs. $4.0 \%, p=0.005)$ or ${ }^{177}$ Lu-DOTATOC $(9.5 \%$ vs. $1.4 \%$, $p=0.002$ ). Renal toxicity was similar among the treatments. Conclusions Using ${ }^{90} \mathrm{Y}$ and ${ }^{177} \mathrm{Lu}$ might facilitate tailoring radiopeptide therapy and improve survival in patients with neuroendocrine tumors.

Keywords sstr2 - Metabolic therapy · Targeted therapy · Survival

\section{Introduction}

The radiolabeled somatostatin analogues DOTATOC and DOTATATE have been used successfully in receptormediated targeted treatment of neuroendocrine tumors [1]. Somatostatin receptor-targeted radiopeptide therapy uses the therapeutic properties of the radionuclides ${ }^{90} \mathrm{Y}$ and ${ }^{177} \mathrm{Lu}$, with the former delivering high target doses and the latter delivering the dose within a smaller range [2-4]. Although the radioisotopes differ in their physical properties, standard clinical dosing regimens have been established [5]. The widespread practice of single-isotope treatment has limited the development of tailored radiopeptide therapy with the combination of ${ }^{90} \mathrm{Y}$ and ${ }^{177} \mathrm{Lu}$. Moreover, no randomized studies are available to compare the benefits and harms of either radioisotope or their combination.

With availability of both isotopes at our center, our data from large prospective cohort studies in patients with metastasized neuroendocrine tumors suggest the effectiveness of 
${ }^{90}$ Y-DOTATOC treatment [1], longer survival with ${ }^{177} \mathrm{Lu}-$ DOTATOC than with ${ }^{90}$ Y-DOTATOC treatment in selected patient groups [6], and longer survival with combined treatment than with ${ }^{90}$ Y-DOTATOC alone [5].

In this study we compared the effectiveness of treatment with ${ }^{90}$ Y-DOTATOC plus ${ }^{177}$ Lu-DOTATOC or either radiopeptide alone by performing multivariableadjusted individual patient-based survival analyses and competing risk analyses using the complete dataset in all 1,499 patients enrolled in the studies described above, with the aim of establishing a framework for tailored radiopeptide therapy.

\section{Materials and methods}

Patients

Patients were recruited worldwide for the three studies using identical inclusion and exclusion criteria as described previously [1, 5, 6]. Recruitment started in 1997, when WHO 2000 and WHO 2010 classifications had not been established. Tumors were classified into four groups: pancreatic neuroendocrine tumors, rare neuroendocrine tumors, neuroendocrine tumors of unknown origin and carcinoids, defined as ileal neuroendocrine tumors with carcinoid syndrome. The studies were carried out in accordance with good clinical practice guidelines, approved by the local ethics committee for human studies, and written informed consent was obtained from all participants or their legal representatives.

\section{Intervention}

Radiopeptide therapy was administered uniformly across the three studies using standard dosing regimens with 7.4 GBq ${ }^{177} \mathrm{Lu}$-DOTATOC and $3.7 \mathrm{GBq} / \mathrm{m}^{2}{ }^{90} \mathrm{Y}$ DOTATOC. ${ }^{90}$ Y-DOTATOC was available at our treatment center from 1997, while ${ }^{177} \mathrm{Lu}$-DOTATOC and ${ }^{90} \mathrm{Y}$ DOTATOC plus ${ }^{177} \mathrm{Lu}$-DOTATOC were available from 2001. There were no strict criteria for allocating patients to the different treatment forms, as previously described $[5,6]$, and there was no cross-treatment between treatment groups. DOTATOC synthesis, radiolabeling and quality control were performed as previously described $[1,5,6]$. Patient preparation and intratherapeutic somatostatin receptor imaging were performed as previously described $[1,5,6]$. Repeated treatment cycles were performed without an a priori defined upper limit at a minimum interval of 6 weeks. Further cycles were withheld because of progression, permanent toxicity, loss of the ability to travel to the treatment center, or the patient's refusal of further treatment.
Follow-up

Follow-up data were actively obtained from referring physicians, primary practitioners or from the patient, and approved for completeness at the study center. Patients were followed with a minimum frequency of two visits per year in our clinic or the referring institution. Data were collected until the patient's death as described previously $[1,5$, 6]. Acute and long-term adverse events were graded according to the Common Terminology Criteria for Adverse Events version 3.0 of the National Cancer Institute [7]. Renal toxicities were classified according to guidelines of the National Kidney Foundation, where grade 4 and 5 renal toxicity are defined as a glomerular filtration rate $<30$ and $<15 \mathrm{~mL} / \mathrm{min} / 1.73 \mathrm{~m}^{2}$, respectively.

Outcomes and statistical analyses

Multivariable-adjusted individual patient-based survival analyses and competing risk analyses were performed using the complete dataset for all 1,499 patients. The main outcomes of interest were survival and toxicity. Survival was assessed from the time of first radiopeptide treatment to death from any cause. Multivariable Cox regression was used to study predictors of survival and renal toxicity taking into account competing risks. Cox regression models included the following independent variables: gender, age, previous treatment, metastases and tumor uptake score (Supplementary Table S1). Effect estimates are expressed as hazard ratios (HR) with $95 \%$ confidence intervals (CI). Two-sided $p$ values of $<0.05$ were considered to indicate statistical significance.

\section{Results}

Patients

Overall, 2,041 patients were screened for eligibility between February 1997 and February 2010; 130 patients $(6.4 \%)$ were not eligible and 412 patients $(20 \%)$ were eligible but not enrolled (Fig. 1). The remaining 1,499 patients (73\%) were treated with ${ }^{90}$ Y-DOTATOC, ${ }^{177} \mathrm{Lu}$-DOTATOC or ${ }^{90} \mathrm{Y}$ DOTATOC plus ${ }^{177} \mathrm{Lu}$-DOTATOC. Patients were recruited from over 100 centers in 29 countries. Their baseline characteristics are shown in Table 1.

Intervention

A total of 3,653 treatment cycles were administered across the three treatment groups (median number of cycles two, range one to ten). Retreatment was performed due to clinical improvement (in 478 patients, $31.9 \%$ ), posttherapeutic tumor 


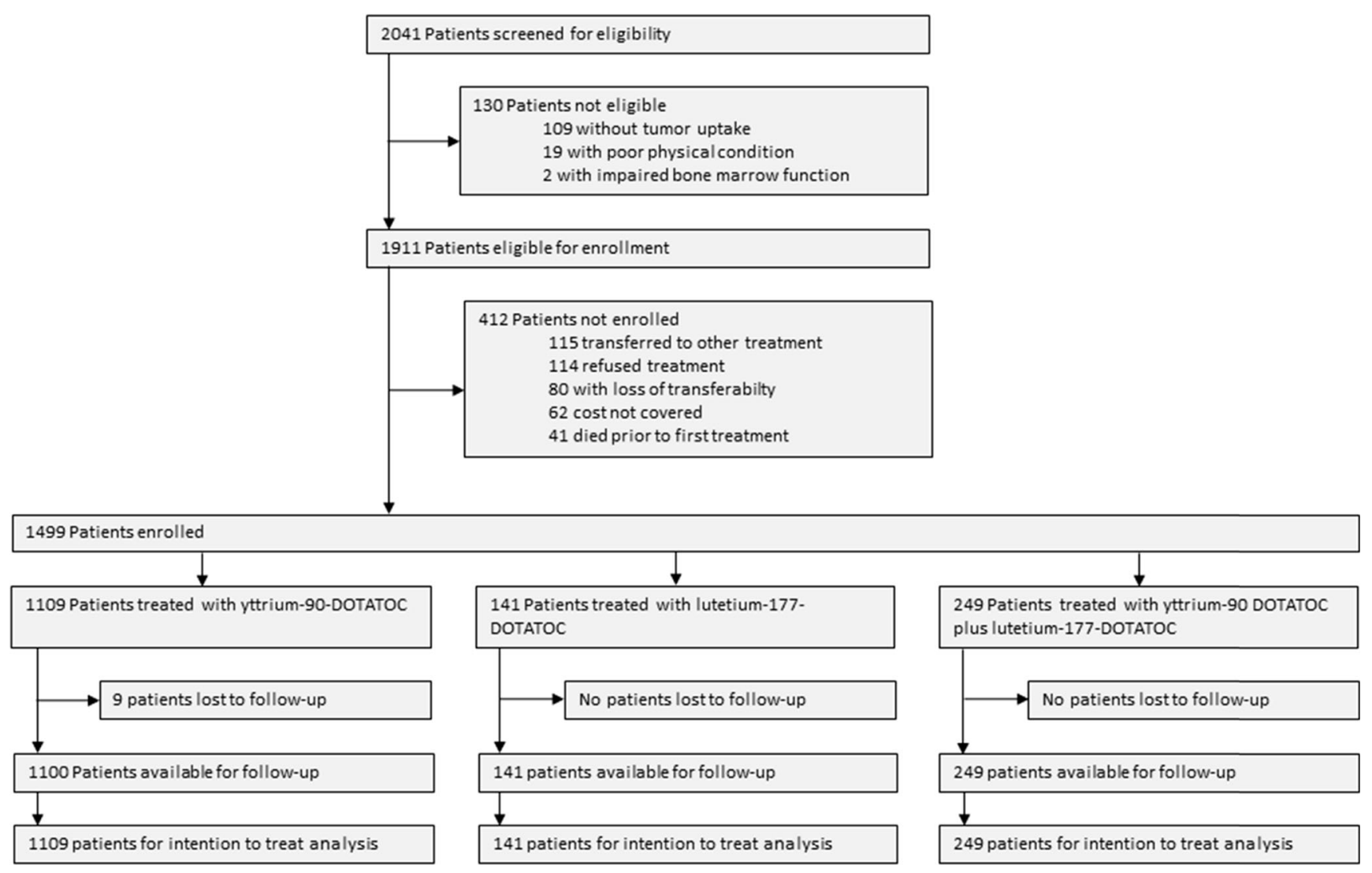

Fig. 1 Trial profile

marker decrease (in 267 patients, $17.8 \%$ ) and/or stabilization or decrease in the sum of the longest diameters of the pretherapeutically detected tumor lesions (in 542 patients, $36.2 \%)$. Specifically, a total of 2,472 cycles were administered in the ${ }^{90}$ Y-DOTATOC group, a 259 in the ${ }^{177} \mathrm{Lu}$ DOTATOC group and a 922 in the ${ }^{90}$ Y-DOTATOC plus ${ }^{177}$ Lu-DOTATOC group (Table 2).

\section{Survival}

Overall, 625 patients $(41.7 \%)$ died, 865 (57.7\%) survived and $9(0.6 \%)$ were not available for follow-up. The median survival from diagnosis was 52.2 months (range $1.1-628.3$ months). The median survival from DOTATOC treatment was 14.5 months (range $1.0-157.6$ months). Patient survival after ${ }^{90}$ Y-DOTATOC, ${ }^{177}$ Lu-DOTATOC and combined treatment is shown in Table 2.

${ }^{90}$ Y-DOTATOC plus ${ }^{177} \mathrm{Lu}$-DOTATOC treatment was associated with longer survival than ${ }^{90} \mathrm{Y}$-DOTATOC (66.1 vs. 47.5 months; $n=1,358 ; p<0.001$; Fig. 2 ) or ${ }^{177} \mathrm{Lu}$-DOTATOC alone (66.1 vs. 45.5 months; $n=390 ; p<0.001$; Fig. 2 ). This observation also applied to patients with multiple metastases, liver metastases, bone metastases or high tumor uptake (Fig. 2) ${ }^{177}$ Lu-DOTATOC was associated with longer survival than ${ }^{90}$ Y-DOTATOC in patients with solitary lesions (HR
0.3 , range $0.1-0.7 ; n=153 ; p=0.005)$, extrahepatic metastases (HR 0.5, range $0.3-0.9 ; n=256 ; p=0.029$ ) and metastases with low uptake (HR 0.1, range $0.05-0.4 ; n=113 ; p=0.001$; Fig. 2). The complete list of survival predictors is shown in Supplementary Table S1.

\section{Toxicities}

There were no significant differences in severe renal toxicity events among the three treatments. During a median follow-up of 11.3 months (range 1.0 - 157.6 months), 130 severe renal toxicity events were observed across the treatment groups, with 89 events in the ${ }^{90}$ Y-DOTATOC group, 13 events in the ${ }^{177} \mathrm{Lu}$-DOTATOC group and 28 events in the ${ }^{90}$ Y-DOTATOC plus ${ }^{177} \mathrm{Lu}$-DOTATOC group (Fig. 3). The median glomerular filtration rate at baseline was $93.8 \mathrm{~mL} / \mathrm{min} / 1.73 \mathrm{~m}^{2}$ (range $30.1-371.5 \mathrm{~mL} / \mathrm{min} / 1.73 \mathrm{~m}^{2}$ ), and decreased by $26.7 \%$ to $68.8 \mathrm{~mL} / \mathrm{min} / 1.73 \mathrm{~m}^{2}$ (range $4.0-259.5 \mathrm{~mL} / \mathrm{min} / 1.73 \mathrm{~m}^{2}$ ) after DOTATOC treatment. In more detail, the decreases were $27.5 \%$ after ${ }^{90}$ Y-DOTATOC treatment, $20.7 \%$ after ${ }^{177} \mathrm{Lu}-$ DOTATOC treatment, and $35.3 \%$ after ${ }^{90}$ Y-DOTATOC plus ${ }^{177} \mathrm{Lu}$-DOTATOC treatment.

${ }^{90}$ Y-DOTATOC induced higher rates of hematotoxicity grade $3 / 4$ than combined treatment $(9.5 \%$ vs. $4.0 \%, p=$ $0.005)$ or treatment with ${ }^{177} \mathrm{Lu}$-DOTATOC alone $(9.5 \%$ vs. 
Table 1 Baseline patient characteristics

\begin{tabular}{|c|c|c|c|c|}
\hline Characteristic & & $\begin{array}{l}{ }^{90} \text { Y-DOTATOC } \\
(n=1,109)\end{array}$ & $\begin{array}{l}{ }^{177} \text { Lu-DOTATOC } \\
(n=141)\end{array}$ & $\begin{array}{l}{ }^{177} \mathrm{Lu} \text {-DOTATOC plus } \\
{ }^{90} \mathrm{Y} \text {-DOTATOC }(n=249)\end{array}$ \\
\hline \multirow[t]{2}{*}{ Gender } & Female & $477(43.0 \%)$ & $59(41.8 \%)$ & $98(39.4 \%)$ \\
\hline & Male & $632(57.0 \%)$ & $82(58.2 \%)$ & $151(60.6 \%)$ \\
\hline \multirow[t]{2}{*}{ Age (years) } & Median & 58.9 & 62.4 & 58.9 \\
\hline & Range & $11.2-91.1$ & $14.8-83.4$ & $23.2-79.6$ \\
\hline \multirow[t]{2}{*}{ Disease duration (years) } & Median & 1.9 & 1.4 & 1.5 \\
\hline & Range & $0.1-37.8$ & $0.1-30.8$ & $0.1-51.2$ \\
\hline \multirow[t]{3}{*}{ Pretreatment } & Surgery & $605(54.6 \%)$ & $79(56 \%)$ & $156(62.7 \%)$ \\
\hline & Chemotherapy & $329(29.7 \%)$ & $17(12.1 \%)$ & $62(24.9 \%)$ \\
\hline & Radiation & $143(12.9 \%)$ & $46(32.6 \%)$ & $22(8.8 \%)$ \\
\hline \multirow[t]{3}{*}{ Extent } & Single Metastasis & $110(9.9 \%)$ & $43(30.5 \%)$ & $39(15.7 \%)$ \\
\hline & Liver Metastases & $912(82.2 \%)$ & $71(50.4 \%)$ & $189(75.9 \%)$ \\
\hline & Bone Metastases & $212(19.0 \%)$ & $22(15.6 \%)$ & $28(11.2 \%)$ \\
\hline \multirow[t]{2}{*}{ Creatinine $(\mu \mathrm{mol} / \mathrm{l})$} & Median & 70.0 & 95 & 73 \\
\hline & Range & $22-434$ & $26-585$ & $33-140$ \\
\hline \multirow[t]{3}{*}{ Tumor uptake score } & 1 & $68(6.1 \%)$ & $45(39.1 \%)$ & $25(10.0 \%)$ \\
\hline & 2 & $68(6.1 \%)$ & $22(15.6 \%)$ & $26(10.4 \%)$ \\
\hline & 3 & $973(87.7 \%)$ & $74(52.5 \%)$ & $198(79.5 \%)$ \\
\hline \multirow[t]{4}{*}{ Kidney uptake score } & 0 & $56(5.0 \%)$ & $7(5.0 \%)$ & $6(2.4 \%)$ \\
\hline & 1 & $130(11.7 \%)$ & $9(6.4 \%)$ & $35(14.1 \%)$ \\
\hline & 2 & $259(23.3 \%)$ & $23(16.3)$ & $52(20.9 \%)$ \\
\hline & 3 & $657(59.2 \%)$ & $102(72.3 \%)$ & $156(62.7 \%)$ \\
\hline \multirow[t]{4}{*}{ Histology } & Carcinoid & $479(43.2 \%)$ & $61(43.3 \%)$ & $109(43.8 \%)$ \\
\hline & Pancreatic neuroendocrine tumor & $342(30.8 \%)$ & $26(18.4 \%)$ & $81(32.5 \%)$ \\
\hline & Rare neuroendocrine tumor & $103(9.3 \%)$ & $24(17.0 \%)$ & $22(8.8 \%)$ \\
\hline & Unknown primary & $185(16.7 \%)$ & $30(21.3 \%)$ & $37(14.9 \%)$ \\
\hline
\end{tabular}

$1.4 \%$; $p=0.002$; Table 3). Overall, 1,985 hematotoxicity events occurred in all three groups. Hematotoxicity grade 1 occurred in 1,396 patients, grade 2 in 434 patients, grade 3 in 123 patients and grade 4 in 32 patients.

Table 2 Results according to treatment group

\begin{tabular}{|c|c|c|c|c|}
\hline & & $\begin{array}{l}{ }^{90} \text { Y-DOTATOC } \\
(n=1,109)\end{array}$ & 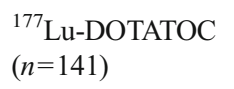 & $\begin{array}{l}{ }^{177} \text { Lu-DOTATOC plus } \\
{ }^{90} \text { Y-DOTATOC }(n=249)\end{array}$ \\
\hline \multirow[t]{2}{*}{ Cycles } & Median & 2 & 2 & 3 \\
\hline & Range & $1-10$ & $1-5$ & $3-8$ \\
\hline \multirow[t]{2}{*}{ Cumulative activity (GBq) } & Mean & 5.9 & 13.5 & 12.4 \\
\hline & SD & 3.0 & 6.5 & 4.9 \\
\hline \multirow[t]{3}{*}{ Response } & Clinic & $329(29.7 \%)$ & $36(25.5 \%)$ & $113(45.4 \%)$ \\
\hline & Biomarkers & $172(15.5 \%)$ & $23(16.3 \%)$ & $72(28.9 \%)$ \\
\hline & Tumor size & $436(39.3 \%)$ & $34(24.1 \%)$ & $134(53.8 \%)$ \\
\hline \multirow[t]{3}{*}{ Survival } & Died & $491(44.3 \%)$ & $48(34.0 \%)$ & $86(34.5 \%)$ \\
\hline & Survived & $609(54.9 \%)$ & $93(66.0 \%)$ & $163(65.5 \%)$ \\
\hline & Lost to follow-up & $9(0.8 \%)$ & 0 & 0 \\
\hline \multirow[t]{2}{*}{ Survival from diagnosis (months) } & Median & 48.2 & 42.9 & 64.9 \\
\hline & Range & $1.1-471.9$ & $3.0-378.5$ & $6.0-628.3$ \\
\hline \multirow[t]{2}{*}{ Survival from DOTATOC treatment (months) } & Median & 12.3 & 9.0 & 35.8 \\
\hline & Range & $1.0-157.6$ & $0.1-80.1$ & $2.4-140.0$ \\
\hline
\end{tabular}


Table 3 Hematotoxicities

\begin{tabular}{lllll}
\hline Hematotoxicity grade & Hematotoxicity & ${ }^{90}$ Y-DOTATOC & ${ }^{177}$ Lu-DOTATOC & ${ }^{90}$ Y-DOTATOC plus ${ }^{177}$ Lu-DOTATOC \\
\hline 1 & Leukopenia & $130(11.7 \%)$ & $8(5.7 \%)$ & $37(14.9 \%)$ \\
& Anemia & $563(50.7 \%)$ & $58(41.1 \%)$ & $143(57.4 \%)$ \\
& Thrombocytopenia & $321(28.9 \%)$ & $27(19.1 \%)$ & $109(43.7 \%)$ \\
2 & Leukopenia & $160(14.4 \%)$ & $12(8.5 \%)$ & $46(18.5 \%)$ \\
& Anemia & $114(10.3 \%)$ & $14(9.9 \%)$ & $4(1.6 \%)$ \\
& Thrombocytopenia & $65(5.9 \%)$ & $5(3.5 \%)$ & $7(2.8 \%)$ \\
3 & Leukopenia & $61(5.5 \%)$ & $0(0 \%)$ & $0(0 \%)$ \\
& Anemia & $10(0.9 \%)$ & $0(0 \%)$ & $0(1.2 \%)$ \\
4 & Thrombocytopenia & $40(3.6 \%)$ & $2(1.4 \%)$ & $0(0 \%)$ \\
& Leukopenia & $6(0.5 \%)$ & $0(0 \%)$ & $1(0.4 \%)$ \\
\hline
\end{tabular}

All toxicities were scored according to the NCI criteria.

\section{Discussion}

Strengths and limitations

The availability of ${ }^{90} \mathrm{Y}$ and ${ }^{177} \mathrm{Lu}$ at our institution allowed direct comparisons of radiopeptide therapy with the two isotopes - both individually and combined. Although not randomized, this study represents the largest cohort treated with ${ }^{90}$ Y-DOTATOC, ${ }^{177}$ Lu-DOTATOC or combination therapy, with the longest patient follow-up available. This large cohort enabled us to adjust all statistical analyses to relevant covariables, including tumor uptake. Follow-up laboratory tests and imaging studies were performed at the referral centers, either with CT or MRI. This practice was feasible for worldwide referrals, but did not permit analyses with the intermediate endpoint of RECIST-defined response. Thus, all efficacy analyses were performed with the endpoint of overall survival. Due to worldwide patient referrals, data on treatments received after DOTATOC are also limited. Finally, even though ${ }^{90}$ Y-DOTATOC and ${ }^{177}$ Lu-DOTATOC have different therapeutic efficacies, the standard dosing regimens that have been established for the two isotopes were applied in all patients.

\section{Comparison with other studies}

Most previous studies have compared ${ }^{90}$ Y-DOTATOC and

${ }^{177}$ Lu-DOTATATE, a comparison that may fail to correctly evaluate differences in efficacy and toxicity given the unique receptor affinities of the two different ligands. Smaller studies with ${ }^{90}$ Y-DOTATOC and ${ }^{177}$ Lu-DOTATA TE have suggested lower renal toxicity rates with ${ }^{177} \mathrm{Lu}-$ based radiopeptide treatment $[8,9]$. Contrary to this, the present study found similar renal toxicity rates across all three DOTATOC treatment groups. We also found higher rates of hematotoxicity with ${ }^{90} \mathrm{Y}$-DOTATOC than with ${ }^{177}$ Lu-DOTATOC, which is comparable with previously reported results [10].

Large randomized controlled trials to evaluate somatostatin receptor-targeted radiopeptide therapy with ${ }^{90} \mathrm{Y},{ }^{177} \mathrm{Lu}$ and their combination are currently not available. Thus, the current study is the best available comparison of the most frequently used radioisotopes in radiopeptide therapy.

\section{Implications}

The present study identified patient stratification algorithms by which both isotopes can be tailored, indicating that treatment centers should aim to utilize both ${ }^{90} \mathrm{Y}$ and ${ }^{177}$ Lu. For example, patients with multiple metastases, liver metastases, bone metastases and high tumor uptake may benefit from combination therapy with ${ }^{90} \mathrm{Y}$ DOTATOC plus ${ }^{177}$ Lu-DOTATOC as compared to either treatment alone. Patients with solitary lesions, extrahepatic metastases and metastases with low uptake may benefit from ${ }^{177} \mathrm{Lu}$-DOTATOC treatment rather than ${ }^{90} \mathrm{Y}$ DOTATOC treatment. In patients in whom hematotoxicity is of particular concern, ${ }^{90}$ Y-DOTATOC alone should be avoided. Furthermore, our results suggest that concern over renal toxicity should not preclude a treatment regimen that includes ${ }^{90}$ Y-DOTATOC.

Additional advances in tailoring the treatment strategy might emerge from trials incorporating histological tumor characteristics. Nevertheless, barriers in conducting randomized controlled trials in the field of somatostatinbased radiopeptide therapy are present, considering the large required sample size and length of follow-up that 
such studies would require. Patient randomization in a study evaluating ${ }^{90} \mathrm{Y}$-DOTATOC would be difficult, as treating a patient with preexisting hematotoxicity with ${ }^{90}$ Y-DOTATOC alone would not be ethical in light of our results and others. However, multi-institutional collaborations are feasible and should be established to advance our understanding of how these tools can be better applied toward individualized patient care.

\section{Conclusions}

The present data indicate no a priori superiority of ${ }^{90} \mathrm{Y}$ based over ${ }^{177} \mathrm{Lu}$-based radiopeptide therapy. However, utilizing both ${ }^{90} \mathrm{Y}$ and ${ }^{177} \mathrm{Lu}$ in adapting a treatment regimen to an individual patient may result in an improved clinical outcome.

Authors' contributions M.A.W. conceived of the study. P.R. designed the study. H.R.M. developed the test drug. J.M.B. administered the test drug. P.R., N.M., P.B., R.D. and M.B. collected and analyzed the data. P.R. and R.D. drafted the first manuscript. M.A.W. supervised study design, data collection, data analysis and writing of the final manuscript.

Acknowledgments We are grateful to our nursing, laboratory and technical staff for their support in patient care, preparation of radiopharmaceuticals and acquisition of scans.

\section{Conflicts of interest None.}

Ethical approval All procedures performed in studies involving human participants were in accordance with the ethical standards of the institutional and national research committee and with the 1964 Helsinki declaration and its later amendments or comparable ethical standards.

Informed consent Written informed consent was obtained from all participants or their legal representatives.

\section{References}

1. Imhof A, Brunner P, Marincek N, Briel M, Schindler C, Rasch H, et al. Response, survival, and long-term toxicity after therapy with the radiolabeled somatostatin analogue [90Y-DOTA]-TOC in metastasized neuroendocrine cancers. J Clin Oncol. 2011;29(17):2416-23. doi:10.1200/JCO.2010.33.7873.

2. Cremonesi M, Ferrari M, Bodei L, Tosi G, Paganelli G. Dosimetry in peptide radionuclide receptor therapy: a review. J Nucl Med. 2006;47(9):1467-75.

3. De Jong M, Valkema R, Jamar F, Kvols LK, Kwekkeboom DJ, Breeman WA, et al. Somatostatin receptor-targeted radionuclide therapy of tumors: preclinical and clinical findings. Semin Nucl Med. 2002;32(2):133-40. doi:10.1053/snuc.2002.31027.

4. Oyen WJ, Bodei L, Giammarile F, Maecke HR, Tennvall J, Luster M, et al. Targeted therapy in nuclear medicine - current status and future prospects. Ann Oncol. 2007;18(11):1782-92. doi:10.1093/annonc/mdm111.

5. Villard L, Romer A, Marincek N, Brunner P, Koller MT, Schindler C, et al. Cohort study of somatostatin-based radiopeptide therapy with [(90)Y-DOTA]-TOC versus [(90)Y-DOTA]-TOC plus [(177)LuDOTA]-TOC in neuroendocrine cancers. J Clin Oncol. 2012;30(10):1100-6. doi:10.1200/JCO.2011.37.2151.

6. Romer A, Seiler D, Marincek N, Brunner P, Koller MT, Ng QK, et al. Somatostatin-based radiopeptide therapy with [177Lu-DOTA]-TOC versus [90Y-DOTA]-TOC in neuroendocrine tumours. Eur J Nucl Med Mol Imaging. 2014;41(2):214-22. doi:10.1007/s00259-013-2559-8.

7. Levey AS, Stevens LA, Schmid CH, Zhang YL, Castro 3rd AF, Feldman HI, et al. A new equation to estimate glomerular filtration rate. Ann Intern Med. 2009;150(9):604-12.

8. Bodei L, Cremonesi M, Ferrari M, Pacifici M, Grana CM, Bartolomei $\mathrm{M}$, et al. Long-term evaluation of renal toxicity after peptide receptor radionuclide therapy with 90Y-DOTATOC and 177Lu-DOTATATE: the role of associated risk factors. Eur J Nucl Med Mol Imaging. 2008;35(10):1847-56. doi:10.1007/s00259-008-0778-1.

9. Kashyap R, Jackson P, Hofman MS, Eu P, Beauregard JM, Zannino $\mathrm{D}$, et al. Rapid blood clearance and lack of long-term renal toxicity of 177Lu-DOTATATE enables shortening of renoprotective amino acid infusion. Eur J Nucl Med Mol Imaging. 2013;40(12):1853-60. doi: 10.1007/s00259-013-2504-x.

10. Sierra ML, Agazzi A, Bodei L, Pacifici M, Arico D, De Cicco C, et al. Lymphocytic toxicity in patients after peptide-receptor radionuclide therapy (PRRT) with 177Lu-DOTATATE and 90Y-DOTATOC. Cancer Biother Radiopharm. 2009;24(6):659-65. doi:10.1089/cbr.2009.0641. 